\title{
A meta-analysis comparing hepatic arterial infusion chemotherapy and sorafenib for advanced hepatocellular carcinoma
}

\author{
Leye Yan, Junqing Lin, Kun Ke, Zhengzhong Wu, Jingyao Huang, Ning Huang, Weizhu Yang \\ Interventional Radiology Department, Fujian Medical University Union Hospital, Fuzhou, China \\ Contributions: (I) Conception and design: L Yan, W Yang; (II) Administrative support: Z Wu, J Huang, N Huang; (III) Provision of study materials \\ or patients: J Lin, K Ke; (IV) Collection and assembly of data: L Yan, J Lin, K Ke; (V) Data analysis and interpretation: All authors; (VI) Manuscript \\ writing: All authors; (VII) Final approval of manuscript: All authors. \\ Correspondence to: Weizhu Yang. Fujian Medical University Union Hospital, No. 29, Xinquan Road, Gulou District, Fuzhou 350001, China. \\ Email: ywzjn2012@163.com.
}

Background: The majority of patients with hepatocellular carcinoma (HCC) are diagnosed in an advanced stage. Although sorafenib is recommended as the standard treatment for advanced HCC, its efficacy is limited. In some studies, hepatic arterial infusion chemotherapy has demonstrated a significant therapeutic benefit for advanced HCC compared with sorafenib. We systematically evaluated and compared the efficacy and safety of hepatic arterial infusion chemotherapy and sorafenib for advanced HCC.

Methods: A systematic search of PubMed, Embase, Web of Science and the Cochrane Library up to December 31, 2020 was conducted. Study outcomes included overall survival (OS), progression-free survival (PFS), objective response rate (ORR), disease control rate (DCR), and adverse effects. The hazard ratio and odds ratio with $95 \%$ confidence intervals $(\mathrm{CI})$ were used to measure the pooled effect.

Results: Eighteen retrospective or prospective cohort studies and one prospective controlled study were included, with 1,339 patients treated with hepatic arterial infusion chemotherapy (HAIC) and 1,060 patients treated with sorafenib. We found that hepatic arterial infusion chemotherapy was superior to sorafenib in terms of OS [hazard ratio (HR): 0.66, 95\% CI: 0.46-0.95, P=0.027], PFS (HR: 0.55, 95\% CI: 0.44-0.69, $\mathrm{P}<0.001$ ), ORR [assessed using Response Evaluation Criteria in Solid Tumors (RECIST): OR: 9.02, 95\% CI: 6.01-13.53, $\mathrm{P}<0.001$; assessed using modified RECIST: odds ratio (OR): 3.71, 95\% CI: 1.92-7.16, $\mathrm{P}<0.001$ ], and DCR (assessed using RECIST: OR: 2.31, 95\% CI: 1.40-3.83, P=0.001; assessed by modified RECIST: OR: 2.28, 95\% CI: 1.22-4.28, P=0.01). Dermatological adverse effects and hypertension were significantly higher in the sorafenib group for all grades of adverse effects. However, regarding severe adverse effects, hepatic arterial infusion chemotherapy was associated with more frequent leukocytopenia and thrombocytopenia.

Conclusions: Hepatic arterial infusion chemotherapy demonstrated favorable efficacy and safety for advanced HCC compared with sorafenib and should be recommended for suitable patients with advanced HCC.

Keywords: Hepatocellular carcinoma (HCC); meta-analysis; sorafenib; hepatic arterial infusion chemotherapy

Submitted Sep 05, 2021. Accepted for publication Dec 02, 2021.

doi: $10.21037 /$ tcr-21-1839

View this article at: https://dx.doi.org/10.21037/tcr-21-1839 


\section{Introduction}

Liver cancer, which is the seventh most common cancer, has an incidence of 9.5 per 100,000 individuals worldwide, causes 830,000 deaths annually and ranks as the fourth most common cause of cancer-related death (1). Moreover, liver cancer is the second most lethal cancer, with a 5 -year survival of $18.1 \%$ (2). Hepatocellular carcinoma (HCC) represents the majority of primary liver cancers and is a major health concern (3).

Sorafenib was the first oral tyrosine kinase inhibitor recommended as a standard systemic treatment for advanced HCC. In the SHARP trial, the median survival of patients receiving sorafenib was $10.7 v s .7 .9$ months in those receiving placebo (4), but only a 3 -month prolongation of the median survival was noted. Since then, little progress has been made. Another novel multityrosine kinase inhibitor, lenvatinib, merely demonstrated a non-inferior outcome with a median survival time of 13.6 months $v s$. those of 12.3 months in sorafenib group (hazard ratio 0.92) (5). In addition, discontinuation of sorafenib might occur due to severe unacceptable toxicity, such as diarrhea and hand-foot skin reaction (4). Recently, immunotherapies have emerged as a promising treatment for cancer, including liver cancer. Immunotherapies including Nivolumab and Pembrolizumab have been approved for advanced HCC by Food and Drug Administration (FDA) (6). In addition, the combinations of atezolizumab and bevacizumab significantly improve overall survival (OS) as compared with sorafenib. Despite those encouraging results, the adverse effects remained high in both groups. Grade 3 or 4 adverse events occurred in $56.5 \%$ of patients who received atezolizumab-bevacizumab and in $55.1 \%$ of patients who received sorafenib (7). Moreover, patients with history of autoimmune disease, coinfection with hepatitis $\mathrm{B}$ or hepatitis $\mathrm{C}$ virus had always been excluded for immune checkpoint inhibitor trials. Thus, an alternative treatment for advanced HCC is required. Hepatic arterial infusion chemotherapy (HAIC) has been reported to lead to favorable outcomes in terms of either the tumor response rate or survival. HAIC directly delivers chemotherapeutic agents to the artery supplying the HCC, which leads to higher concentrations in the lesions. In theory, this treatment would improve efficacy and reduce systemic toxicity compared with intravenous chemotherapy. Although previous meta-analyses conducted by Zhuang et al. support the use of HAIC for advanced HCC with respect to both tumor response rate and survival, the heterogeneity among the included studies remained relatively high (8). In addition, severe adverse events were not assessed separately. Moreover, additional studies have been published since Zhuang's meta-analyses were conducted. The purposes of this meta-analysis were to provide updates on the efficacy and safety of HAIC versus sorafenib for advanced HCC and to perform sensitivity and subgroup analyses to evaluate the source of heterogeneity. We present the following article in accordance with the PRISMA reporting checklist (available at https://tcr. amegroups.com/article/view/10.21037/tcr-21-1839/rc).

\section{Methods}

\section{Study protocol}

We followed the Preferred Reporting Items for Systematic Review and Meta-Analyses (PRISMA) guidelines (9) and preregistered this meta-analysis in the International Prospective Register of Systematic Reviews (PROSPERO; CRD42021231808). A systematic search of PubMed, Embase, Web of Science and the Cochrane Library up to December 31, 2020 was conducted. The search used the terms "hepatic arterial infusion chemotherapy", "sorafenib", and "hepatocellular carcinoma". The language was limited to English, and unpublished articles were not searched [see Supplementary file (Appendix 1) for details]. References of retrieved articles were further screened to find potential related literature.

\section{Selection criteria}

Selection of the literature was performed independently by two investigators (LYY and JQL). Discrepancies were resolved through consensus reached by the two investigators; otherwise, they were arbitrated by a third investigator (KK).

Studies met the following inclusion criteria: (I) confirmation of advanced HCC; (II) inclusion of a treatment group receiving HAIC and a control group receiving sorafenib; (III) data on OS, progression-free survival (PFS), objective response rate (ORR), and disease control rate (DCR); (IV) trials described as randomized controlled trials (RCTs) or retrospective comparative studies. The exclusion criteria included: (I) patients receiving other treatments instead of HAIC and sorafenib or a combination of HAIC and sorafenib; (II) lack of a control group; (III) letters, abstracts, case reports, and studies that did not provide detailed baseline characteristics and outcomes. 


\section{Data extraction}

Data extraction was performed independently by two investigators (LYY and JQL) according to a standardized method. Data collected included (I) author, year of publication, country, trial design, and sample size; (II) baseline characteristics and tumor characteristics; (III) and hazard ratios and $95 \%$ confidence intervals of OS and PFS reported in the studies or derived from survival curves. Data also included (IV) tumor response outcomes, such as complete response (CR), partial response (PR), stable disease (SD), and progressive disease (PD), which were assessed using the Response Evaluation Criteria in Solid Tumors (RECIST) or the modified Response Evaluation Criteria in Solid Tumors (mRECIST) $(10,11)$. The ORR was defined as the sum of CR and PR, while the DCR was defined as the sum of CR, PR, and SD. (V) Adverse effects, which were categorized based on the National Cancer Institute's Common Terminology Criteria for Adverse Events (CTCAE), included hematological and dermatological adverse effects, liver dysfunction, hypertension, and fatigue.

\section{Quality assessment}

The quality of the included studies was assessed using Jadad Scale (12) for randomized trials or the Newcastle-Ottawa Scale (NOS) (13) for cohort studies. The studies with scores greater than or equal to 6 using NOS or that with scores greater than 2 using Jadad Scale (12) were considered to be high-quality.

\section{Statistical analysis}

Hazard ratios (HRs) with $95 \%$ confidence intervals (CIs) were reported directly or obtained from survival curves and calculated using sheets prepared by Tierney et al. (14). Then, HRs with $95 \%$ CIs were calculated for the effects of HACI $v s$. sorafenib on OS and PFS. Odds ratios (ORs) with 95\% CIs were pooled for the ORR, DCR, and AEs. Heterogeneity was qualitatively determined by $\chi^{2}$-based Q-test, and $\mathrm{P}<0.1$ was considered heterogeneous. $\mathrm{I}^{2}$ was used to quantitatively evaluate heterogeneity, and $\mathrm{I}^{2}$ values of $25 \%, 50 \%$, and $75 \%$ refer to low, moderate, and high degrees of heterogeneity, respectively (15). A random effects model was used to pool the estimated effects with significant heterogeneity $\left(\mathrm{P}<0.1\right.$, or $\left.\mathrm{I}^{2}>50 \%\right)$. Otherwise, a fixed effects model was applied. Sensitivity and subgroup analyses were conducted to identify potential sources of heterogeneity. Funnel plots and Begg's and Egger's tests were used to evaluate publication bias. All analyses were conducted using Stata version 12.0 (StataCorp., College Station, TX, USA).

\section{Results}

\section{Literature search}

In all, 789 studies and related references were initially retrieved by database searches, as shown in Figure 1 . Then, 321 duplicate studies were removed. Abstracts and summaries were reviewed to further exclude irrelevant reviews and guidelines, non-comparative studies and case reports. Data from 2 studies overlapped, and conference abstracts without full-text articles were also excluded. Finally, nineteen studies, including 17 retrospective studies (16-32), 1 prospective cohort study (33) and 1 prospective randomized controlled study (34), were included.

\section{Characteristics of the included studies}

The characteristics of the included studies are summarized in Table 1. In all, 1,339 patients were treated with HAIC and 1,060 patients were treated with sorafenib. The publication period ranged from 2011 to 2020 . Among the studies, twelve were conducted in Japan $(16,18-25,28,30,33)$, six were conducted in Korea $(17,26,27,31,32,34)$, and one was conducted in China (29). Cisplatin and 5-fluorouracil (5$\mathrm{FU}$ ) were the most frequently used regimens in HAIC. A dose of $800 \mathrm{mg}$ of sorafenib was administered daily for most patients in the sorafenib group. Dose reduction or discontinuation of sorafenib occurred when severe adverse effects appeared. The quality scores of the cohort studies as determined by the Newcastle-Ottawa Scale ranged from $5-8$. The quality of only one RCT as determined by the Jadad Scale were evaluated as high. In conclusion, most studies [15 (79\%)] were determined to be of high quality, while four studies were determined to be of low quality.

\section{OS}

Eighteen studies (16-24,26-34) reported OS outcomes. Our meta-analysis demonstrated that HAIC was superior to sorafenib in the treatment of advanced HCC with respect to OS (HR: 0.66, 95\% CI: 0.46-0.95; $\mathrm{P}=0.027$ ) (Figure 2). However, high heterogeneity was detected $\left(\mathrm{I}^{2}=89 \%\right.$, $\mathrm{P}<0.001$ ), and a random effects model was applied. A 


\section{Identification of studies via databases and registers}
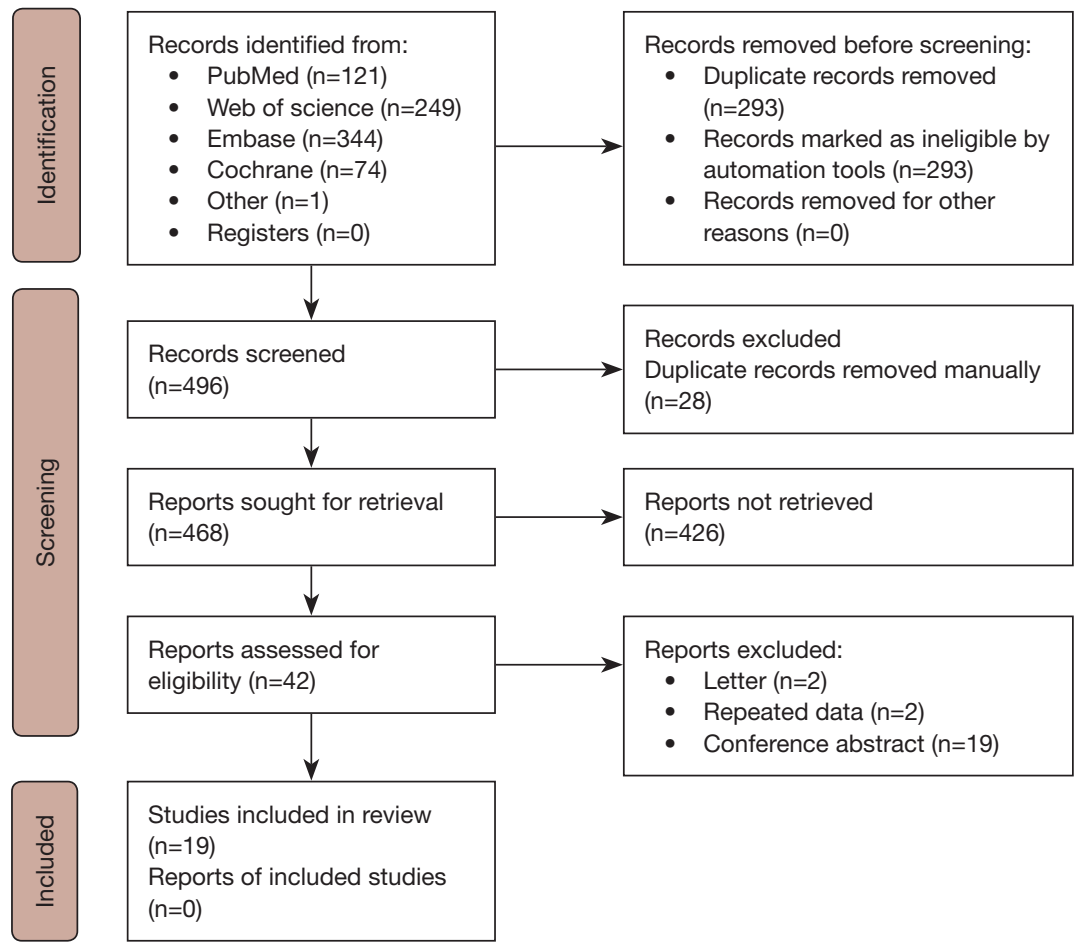

Studies included in review

$(n=19)$

Reports of included studies

$(n=0)$

Figure 1 Flow diagram of the search strategy used in this meta-analysis.

sensitivity study suggested that Lyu's (29) study, in which mFOLFOX was adopted rather than the combination of cisplatin and 5-fluorouracil administered in most cohorts, is the major source of heterogeneity. After Lyu's study was removed, the heterogeneity became more moderate (48.7\%, $\mathrm{P}=0.013)$. No publication bias was detected by visual examination of funnel plots or by Begg's test or Egger's test $(\mathrm{P}=0.256, \mathrm{P}=0.585)$.

\section{PFS}

Thirteen studies $(17,18,20,22-25,27,29,31-34)$ were pooled for the outcome of PFS. Our meta-analysis showed a significant benefit in PFS in favor of HAIC compared with sorafenib (HR: 0.55, 95\% CI: 0.44-0.69; $\mathrm{P}<0.001$ ) (Figure 3). A random effects model was used due to the high heterogeneity between studies $\left(\mathrm{I}^{2}=62.9 \%, \mathrm{P}=0.001\right)$. A sensitivity study suggested that the studies by Lyu (29) and Kondo (22), in which mFOLFOX and cisplatin alone without 5 -fluorouracil were administered as chemotherapy agents, respectively, were sources of heterogeneity. After excluding both studies, the result still favored HAIC (HR:
0.543, 95\% CI: 0.464-0.636, $\mathrm{P}<0.001, \mathrm{I}^{2}=0 \%, \mathrm{P}=0.001$ ). No publication bias was detected by visual examination of funnel plots or by Begg's test or Egger's test $(\mathrm{P}=0.855$, $\mathrm{P}=0.633$ ).

\section{ORR}

Eighteen studies (16-18,20-34) reported the ORR. Among those studies, ten $(16,18,21-25,28,29,33)$ reported an ORR that was evaluated by the RECIST criteria. The heterogeneity was not significant in those studies $\left(\mathrm{I}^{2}=25.4 \%\right.$, $\mathrm{P}=0.210)$. Our meta-analysis showed that HAIC was associated with a higher overall response rate (OR: 9.02, 95\% CI: 6.01-13.53, $\mathrm{P}<0.001$ ) (Figure 4). Begg's test and Egger's test showed no publication bias $(\mathrm{P}=0.858, \mathrm{P}=0.962)$. Ten studies $(17,20,22,26,27,29-32,34)$ were evaluated using the mRECIST criteria. A random effects model was used due to significant heterogeneity among studies $\left(\mathrm{I}^{2}=63.1 \%\right.$, $\mathrm{P}=0.004)$. ORRs were significantly higher in the HAIC group than in the sorafenib group (OR: $3.708,95 \%$ CI: $1.921-7.158, \mathrm{P}<0.001)$. Publication bias was not significant according to Begg's test or Egger's test $(\mathrm{P}=0.474, \mathrm{P}=0.828)$. 


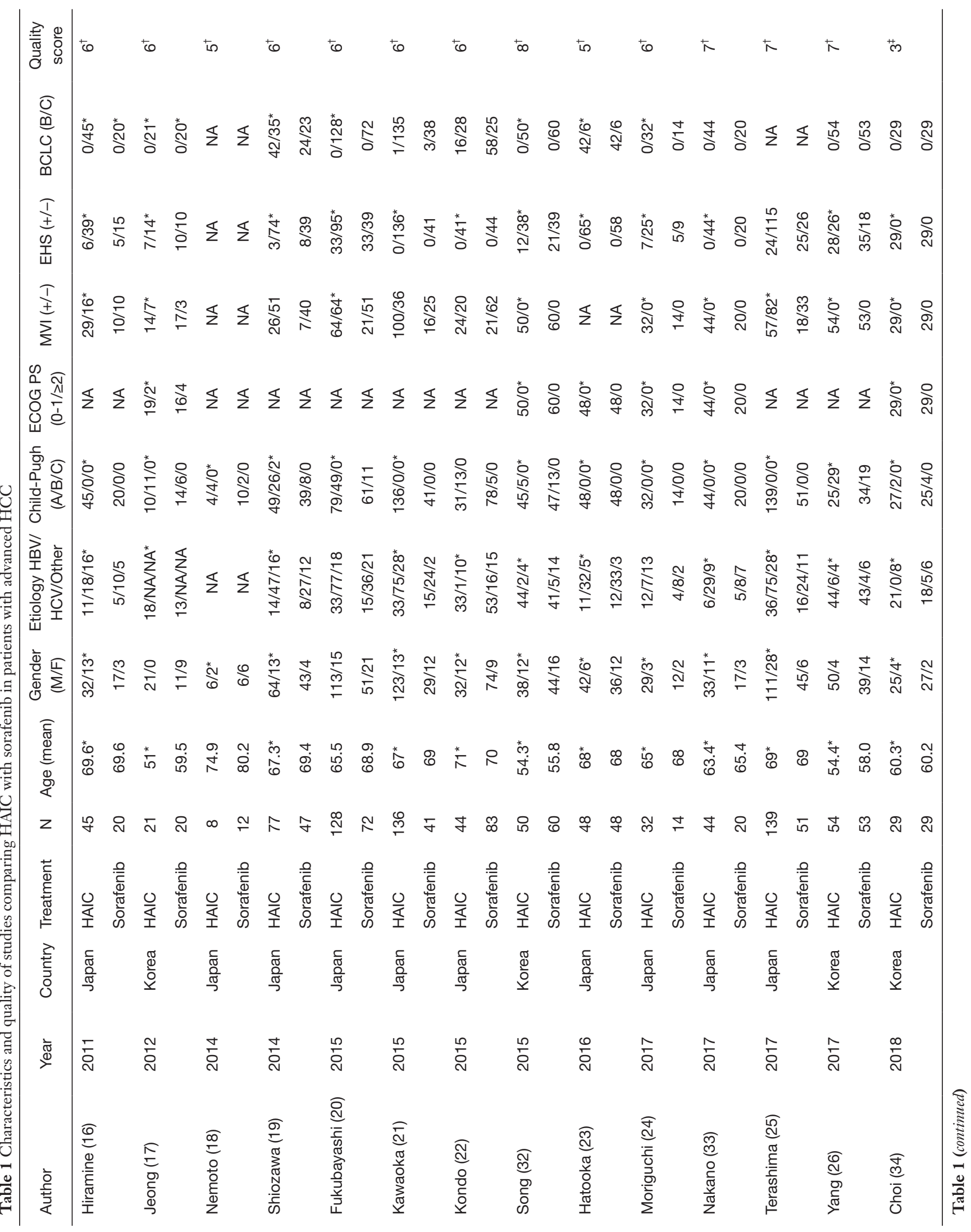




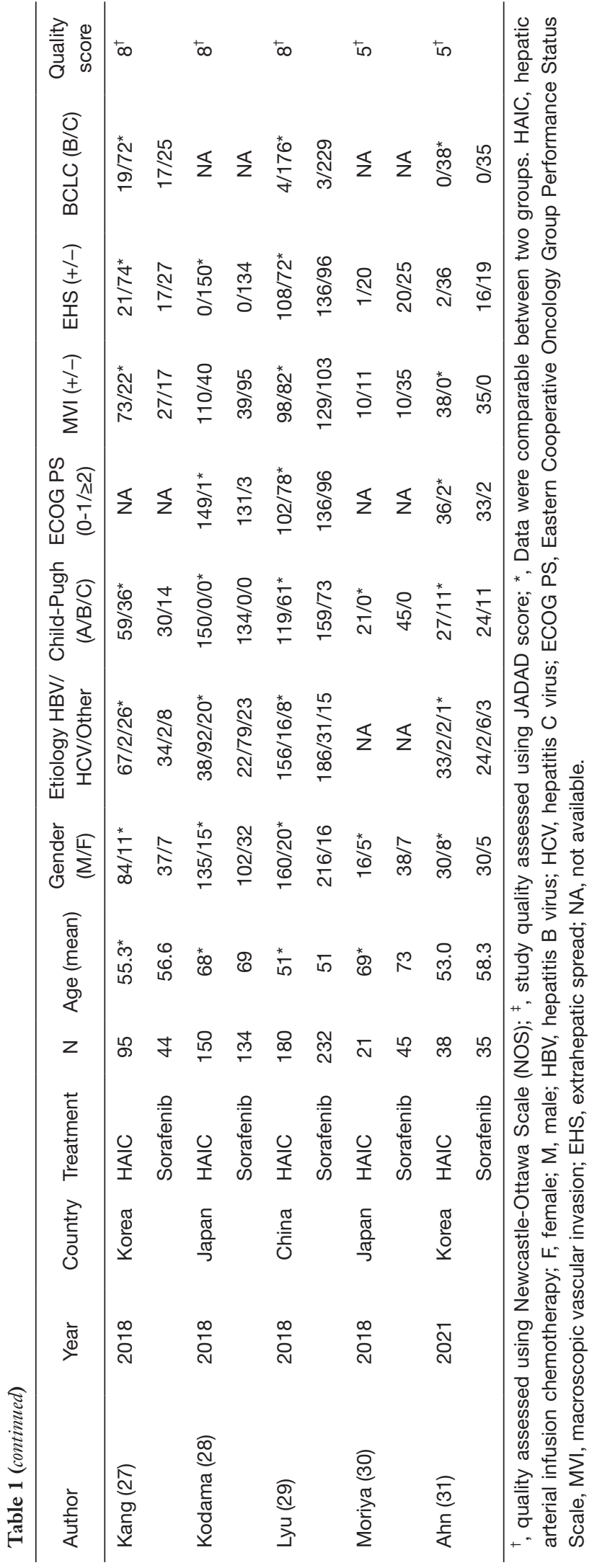

\section{DCR}

A total of eighteen (16-18,20-34) studies reported the DCR. Ten $(16,18,21-25,28,29,33)$ reported overall response rates and were evaluated using the RECIST criteria. The heterogeneity was significant in those studies $\left(\mathrm{I}^{2}=74.3 \%\right.$, $\mathrm{P}<0.001)$. A random effects model found that HAIC led to a higher DCR (OR: 2.31, 95\% CI: $1.40-3.83, \mathrm{P}=0.001$ ) (Figure 5), with no publication bias found (Begg's test $\mathrm{P}=0.721$, Egger's test $\mathrm{P}=0.673)$. In addition, ten studies $(17,20,22,26,27,29-32,34)$ were evaluated using the mRECIST criteria. A random effects model was used due to significant heterogeneity among studies $\left(\mathrm{I}^{2}=82.5 \%\right.$, $\mathrm{P}<0.001)$. The DCR was significantly higher in the HAIC group than in the sorafenib group (OR: 2.284, 95\% CI: 1.218-4.284, $\mathrm{P}=0.01)$. Publication bias was not significant according to either Begg's test or Egger's test $(\mathrm{P}=0.858$, $\mathrm{P}=0.846$ ).

\section{Adverse effects}

Fifteen studies reported adverse effects that were severe or higher than grade 3, while seven studies reported adverse effects of any grade. We compared severe adverse events (SAEs) and AEs of all grades between groups. Our metaanalysis demonstrated that sorafenib was associated with a higher rate of AEs of any grade, including: dermatological adverse effects (OR: 0.021, 95\% CI: $0.007-0.061, \mathrm{P}<0.001$ ) and hypertension-related AEs (OR: 0.032, $95 \% \mathrm{CI}$ : $0.008-0.117, \mathrm{P}<0.001)$, whereas no significant difference was observed in AEs related to leukocytopenia (OR: 6.797, 95\% CI: 0.412-112.235, $\mathrm{P}=0.18$ ), neutropenia (OR: 12.939, 95\% CI: 0.764-219.050, $\mathrm{P}=0.076$ ), anemia (OR: 5.412, 95\% CI: 0.406-72.201, $\mathrm{P}=0.201$ ), thrombocytopenia (OR: 8.557, 95\% CI: 0.701-104.406, $\mathrm{P}=0.093)$, liver dysfunction (OR:1.205, 95\% CI: 0.492-2.951, $\mathrm{P}=0.683$ ), gastrointestinal AEs (OR: 0.508, 95\% CI: 0.169-1.530, $\mathrm{P}=0.229$ ), and fatigue (OR: 0.352, 95\% CI: 0.059-2.109, $\mathrm{P}=0.253$ ).

For severe adverse effects, leukocytopenia (OR: 5.352, 95\% CI: 1.229-23.304, $\mathrm{P}=0.025)$ and thrombocytopenia (OR: $5.319,95 \%$ CI: $1.867-15.159, \mathrm{P}=0.002$ ) were significantly higher in the HAIC group, while liver dysfunction (OR: 0.703, 95\% CI: 0.509-0.971, $\mathrm{P}=0.032$ ), dermatological (OR: 0.067, 95\% CI: 0.029-0.155, P<0.001) and gastrointestinal (OR: 0.153, 95\% CI: 0.082-0.285, $\mathrm{P}<0.001)$ AEs, as well as hypertension (OR: 0.061, 95\% CI: $0.012-0.321, \mathrm{P}=0.001)$ and fatigue (OR: $0.299,95 \%$ CI: $0.163-0.545, \mathrm{P}<0.001)$ were significantly higher in the 


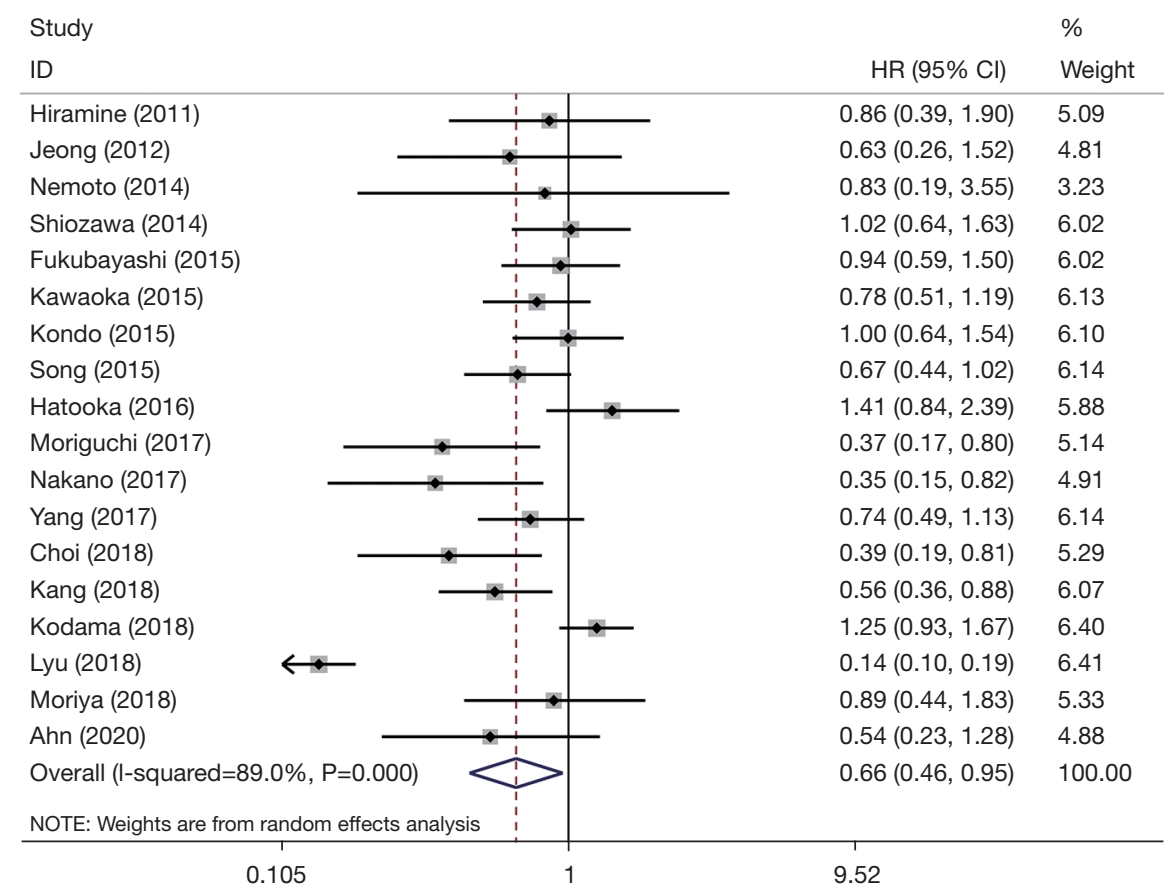

Figure 2 Forest plot of the meta-analysis for overall survival. CI, confidence interval; HR, hazard ratio.

sorafenib group.

\section{Subgroup analysis}

HAIC remained to show a benefit in OS of patients included in studies published after 2017 (HR: 0.496, 95\% CI: $0.263-0.936, \mathrm{P}=0.03)$, those conducted in countries other than Japan (HR: 0.464, 95\% CI: 0.248-0.867, $\mathrm{P}=0.016$ ), those with a small sample size (HR: $0.645,95 \%$ CI: $0.445-0.934, \mathrm{P}=0.02$ ), those that were of high quality (HR: 0.616, 95\% CI: 0.405-0.938, $\mathrm{P}=0.024)$, those with macroscopic vascular invasion (MVI) only (HR: 0.566, 95\% CI: $0.437-0.732, \mathrm{P}<0.001)$, those in which cisplatin + 5-FU or mFOLFOX was used as the HAIC regimen, and those that contained cases with BCLC Stage C only (HR: 0.637, 95\% CI: 0.509-0.797, $\mathrm{P}<0.001)$. Similarly, HAIC remained superior to sorafenib regardless of country, sample size, study quality, and MVI status, except that PFS was significantly higher only in studies published after 2017 (HR: 0.453, 95\% CI: 0.382-0.538, P<0.001) (Table 2).

\section{Discussion}

In this systematic review and meta-analysis, which aimed to compare the efficacy and safety profiles of HAIC and sorafenib for advanced HCC, we found that HAIC was superior to sorafenib in terms of OS, PFS, ORR, and DCR. No significant differences were observed in adverse effects, including leukocytopenia, neutropenia, anemia, thrombocytopenia, liver dysfunction, and gastrointestinal disorders, between the two groups. An exception was for dermatological adverse effects and hypertension, which were significantly higher in the sorafenib group than in the other group with respect to adverse effects of all grades. However, regarding severe adverse effects, HAIC was related to more frequent leukocytopenia and thrombocytopenia, while liver dysfunction, dermatological adverse effects, gastrointestinal disorders, hypertension, and fatigue were significantly higher in the sorafenib group.

Our meta-analysis showed the benefit of HAIC over sorafenib with respect to OS and PFS. Though assessed according to different criteria (i.e., RECIST and mRECIST), the ORR and DCR remained significantly higher in the HAIC group. These results were consistent with those of previous meta-analyses conducted by Zhuang et al. (8). The improvement associated with HAIC in advanced HCC is probably due to direct delivery of chemotherapy agents to lesions and increased concentration. Recently, it has also been proposed that the combination of HAIC with tyrosine kinase inhibitors might 


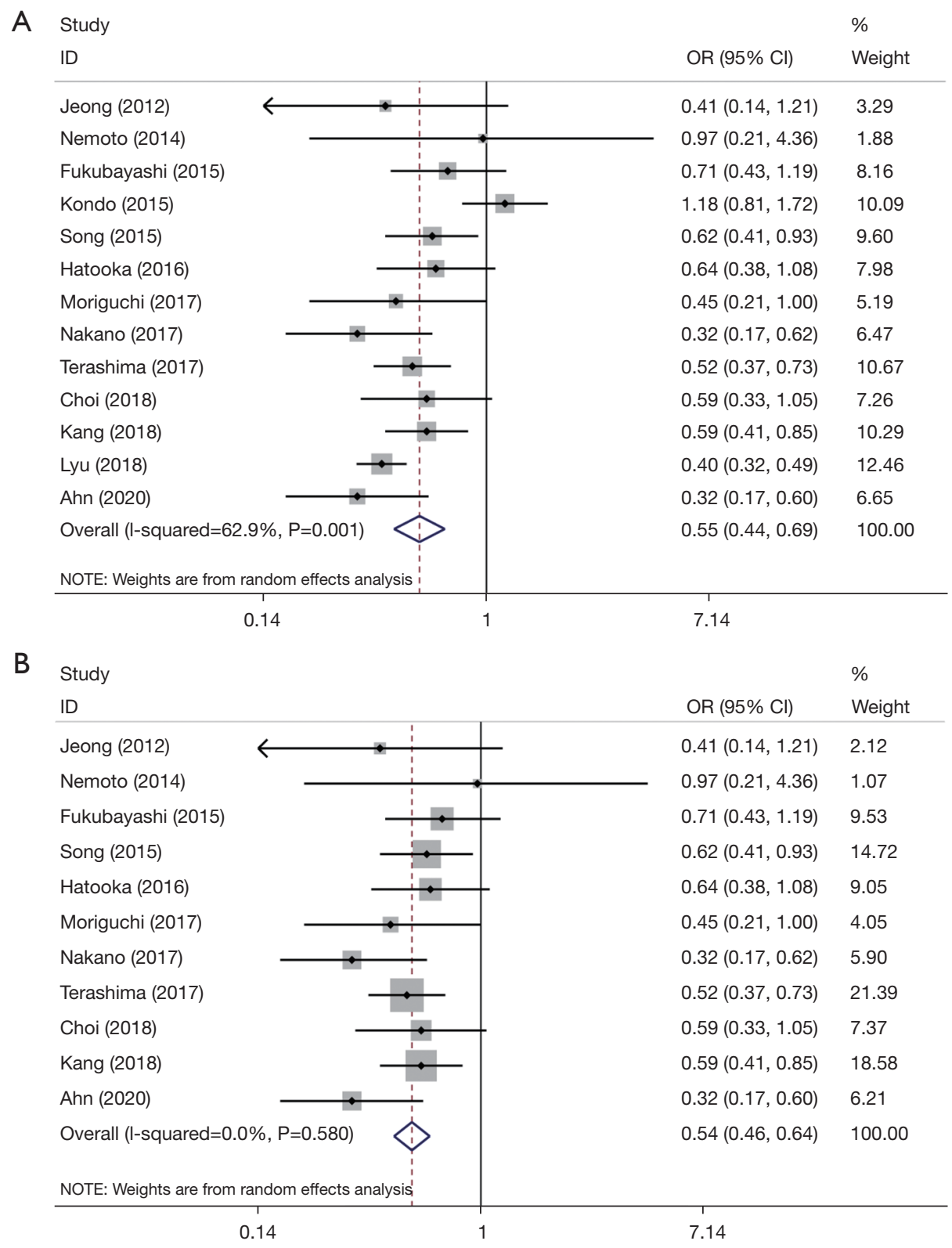

Figure 3 Forest plot of the meta-analysis for progression-free survival. CI, confidence interval; HR, hazard ratio.

benefit patients with advanced HCC through a synergistic anticancer effect. A randomized clinical trial comparing the efficacy and safety of sorafenib plus HAIC $v$ s. sorafenib for HCC with portal vein invasion found that sorafenib plus HAIC of FOLFOX improved OS and had acceptable toxic effects (35). Those clinical benefits of HAIC shown in this trial were consistent with our meta-analysis. Moreover, high heterogeneity was identified in our meta-analysis. Thus, sensitivity studies were performed to identify the source of heterogeneity. Chemotherapy agents administered arterially, such as mFOLFOX and cisplatin alone, explained the majority of the heterogeneity, while other heterogeneity may have resulted from baseline characteristics, study design, sample size, and region. Therefore, high-quality multicenter randomized controlled studies are warranted to further verify this conclusion.

Subgroup analysis demonstrated that patients included in studies published after 2017, those conducted in Korea and China, high-quality studies, studies that included cases with MVI only, those in which cisplatin + 5-FU or mFOLFOX 


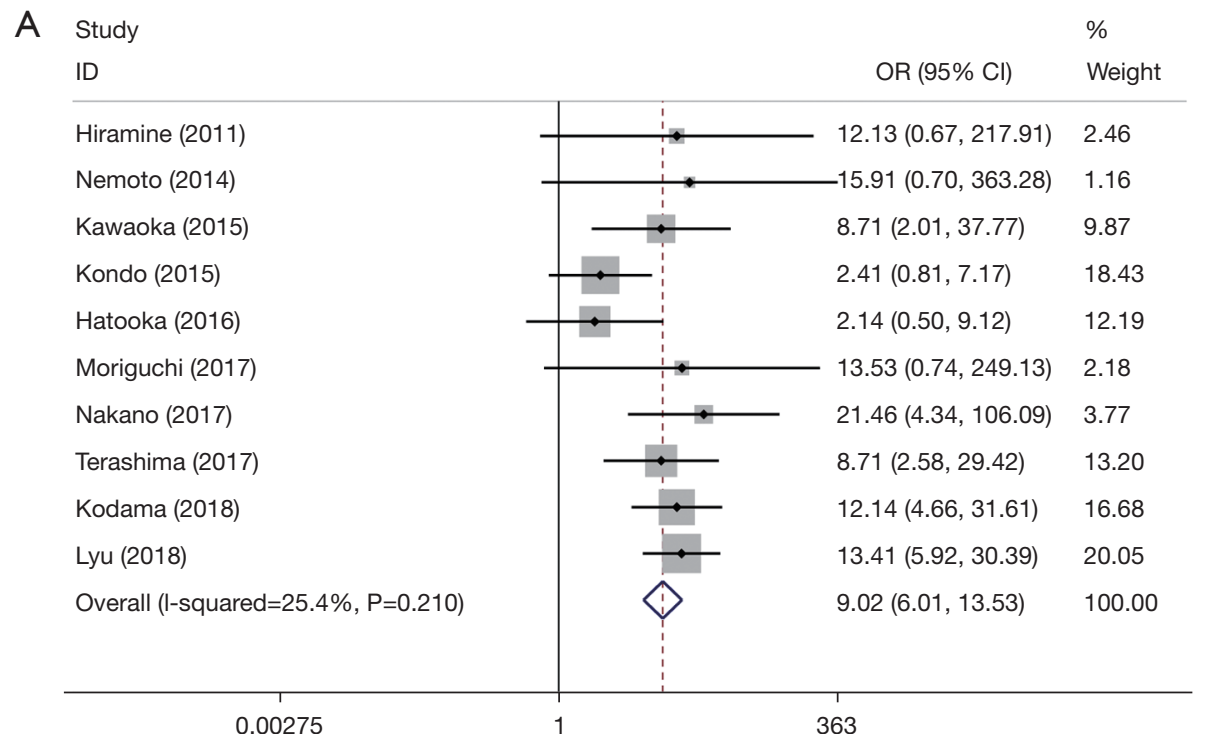

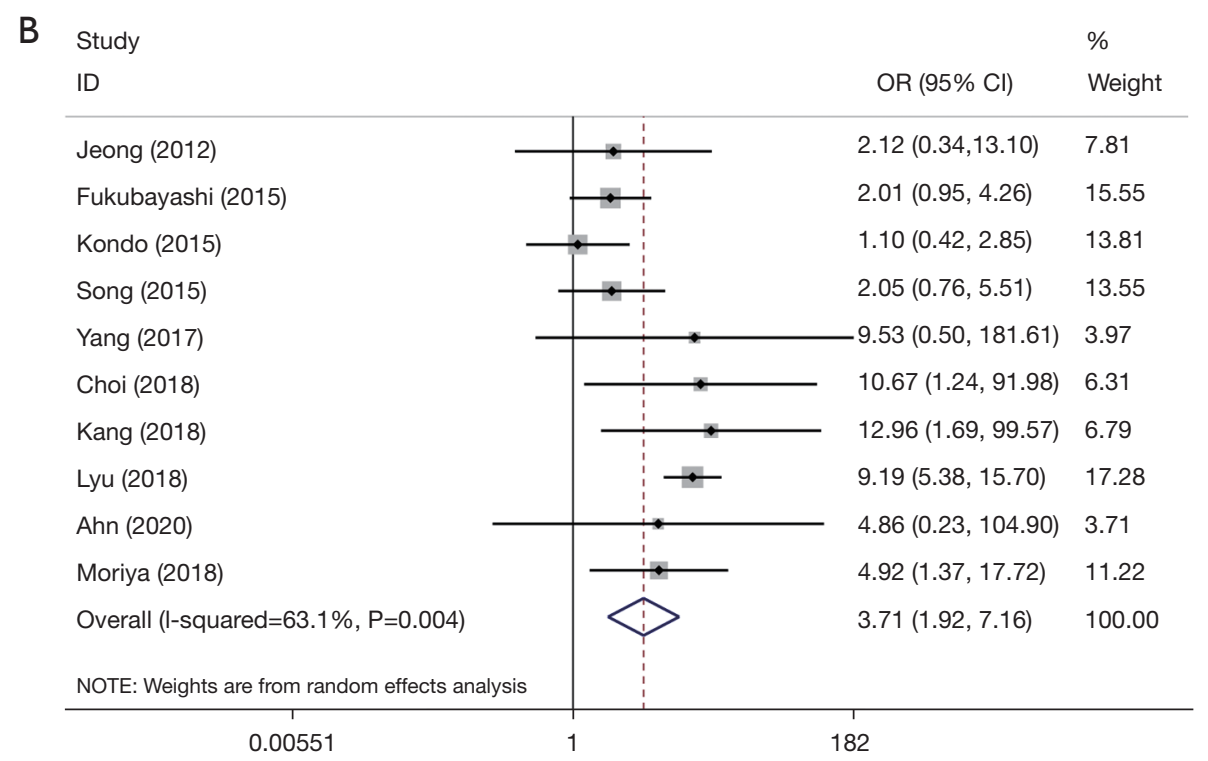

Figure 4 Forest plot of the meta-analysis for objective response rate, as assessed by the RECIST (A) and mRECIST criteria (B). CI, confidence interval; OR, odds ratio; RECIST, Response Evaluation Criteria in Solid Tumors.

were used as the HAIC regimen, and those with BCLC Stage $\mathrm{C}$ only had significantly better OS. The reason that patients in studies conducted in Korea and China had better OS than those included in studies conducted in Japan could be due to the reasons discussed below. (I) Different underlying etiologies of HCC. Studies conducted in Japan tend to include a greater proportion of patients with hepatitis $\mathrm{C}$ virus infections than those conducted in Korea and China. (II) Different HAIC procedures and chemotherapy regimens. In addition, these results indicated that cisplatin $+5-\mathrm{FU}$ or FOLFOX might be a preferred chemotherapy agent. Moreover, patients with advanced HCC with macrovascular invasion had better OS when treated with HAIC than when treated with sorafenib. This result was consistent with that reported in the systematic review by Liu et al., which demonstrated that HAIC is superior to sorafenib in HCC with portal vein thrombosis, especially in HCC with types III-IV portal vein thrombosis (36).

Adverse effects of HAIC were mostly related to chemotherapy and included leukocytopenia, neutropenia, 


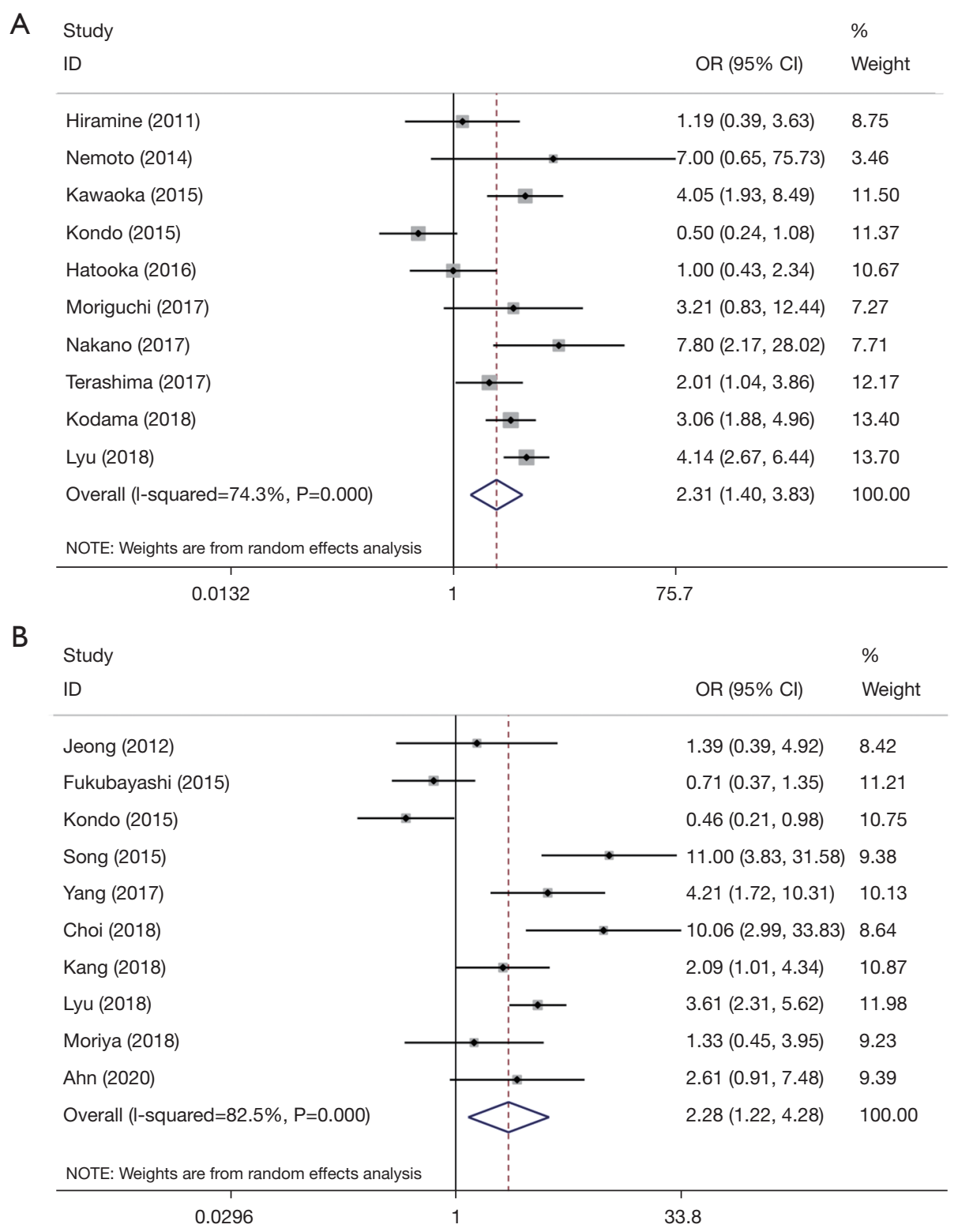

Figure 5 Forest plot of the meta-analysis for disease-control rate, as assessed by the RECIST (A) and mRECIST criteria (B). CI, confidence interval; OR, odds ratio; RECIST, Response Evaluation Criteria in Solid Tumors.

anemia, and thrombocytopenia. Our meta-analysis did not find a significant difference between the two groups in terms of adverse effects of all grades, which was consistent with the study by Zhuang et al. However, the meta-analysis conducted by Zhuang et al. did not pool severe adverse effects (8). Our study found a higher incidence of severe hematological disorders, including leukocytopenia and thrombocytopenia, in the HAIC group. Hence, patients who receive HAIC should be monitored by repeated blood tests. Sorafenib was associated with a greater incidence of liver dysfunction, dermatological adverse effects, gastrointestinal disorders, hypertension, and fatigue than HAIC. In the SHARP trial (4), diarrhea (39\%), hand-foot skin reaction $(21 \%)$, fatigue (22\%), and hypertension (5\%) were reported as the most frequent adverse effects, which was consistent with the findings for sorafenib.

Although our meta-analysis demonstrated that HAIC is superior to sorafenib for advanced HCC, multiple limitations should be considered. First, among the included studies, only one was a randomized controlled study, 
Table 2 Subgroup analysis of overall survival and progression-free survival

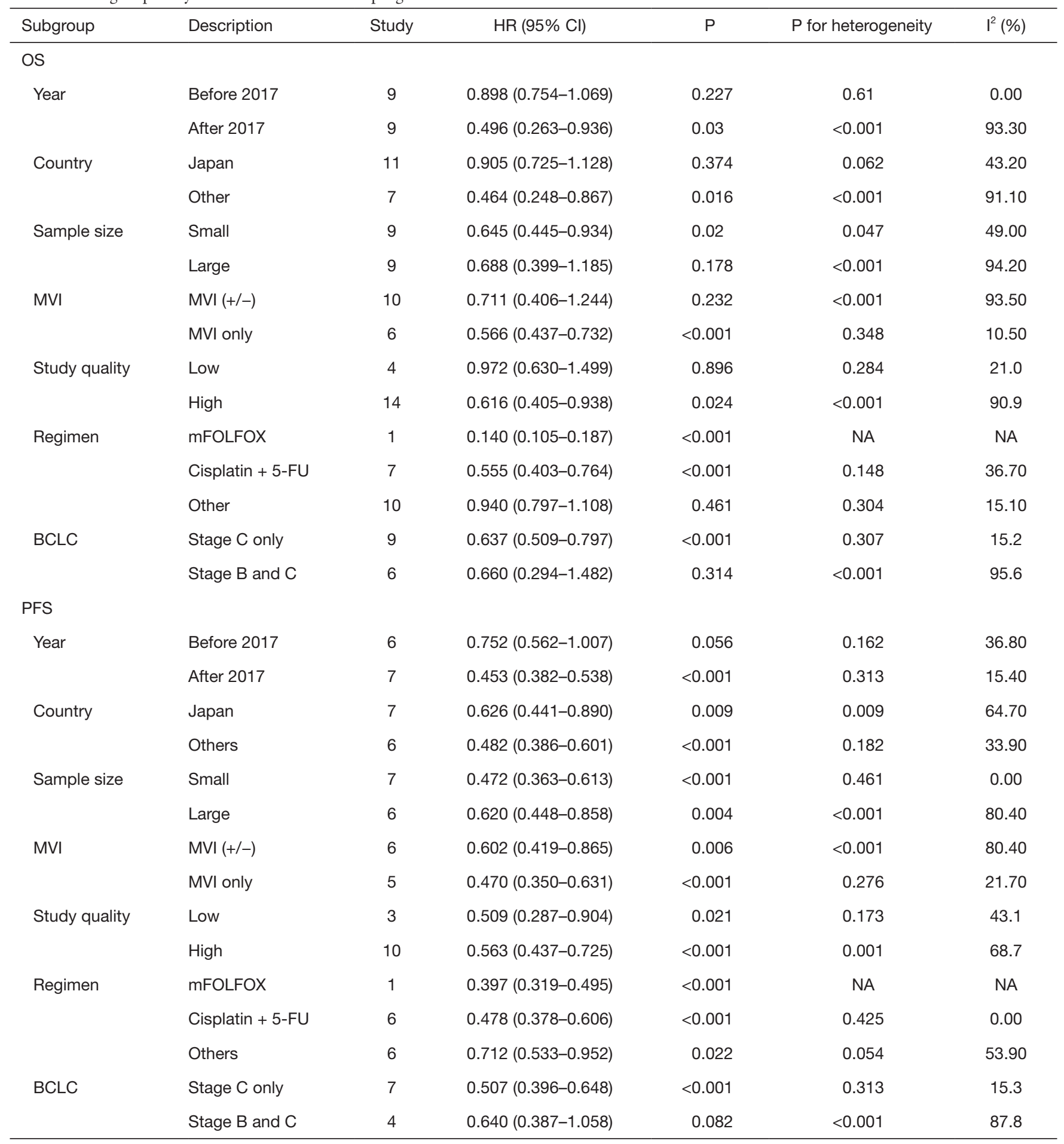

OS, overall survival; PFS, progression-free survival; 5-FU, 5-flurouracil; MVI, macroscopic vascular invasion; HR, hazard ratio; NA, not available. 
whereas the other 18 were retrospective or prospective cohort studies, which could increase selection bias. Second, all studies included were from Asia, and the conclusion may not be applicable to Western populations. Third, the difference in baseline characteristics between the two groups was not totally controlled due to the retrospective study design of most studies. Fourth, the relatively small sample size in some of the included studies may also lead to selection bias or detection bias. Fifth, although cisplatin plus 5-FU was the most common regimen adopted, multiple regimens were sometimes used in a single study. It would be complicated to determine the more effective regimen by subgroup analysis, and therefore, multicenter prospective randomized controlled studies are needed to further verify the outcomes.

\section{Conclusions}

This study demonstrated that HAIC is more effective than sorafenib in terms of OS, PFS, ORR, and DCR. HAIC is associated with a higher incidence of severe hematological adverse effects, whereas sorafenib is associated with higher rates of severe liver dysfunction, dermatological adverse effects, gastrointestinal disorders, hypertension, and fatigue. Further RCTs are required to clarify the optimal chemotherapy regimen and doses of HAIC.

\section{Acknowledgments}

Funding: The study was supported by the Natural Science Foundation of Fujian Province (2019J01162).

\section{Footnote}

Reporting Checklist: The authors have completed the PRISMA reporting checklist. Available at https://tcr. amegroups.com/article/view/10.21037/tcr-21-1839/rc

Peer Review File: Available at https://tcr.amegroups.com/ article/view/10.21037/tcr-21-1839/prf

Conflicts of Interest: All authors have completed the ICMJE uniform disclosure form (available at https://tcr.amegroups. com/article/view/10.21037/tcr-21-1839/coif). The authors have no other conflicts of interest to declare.

Ethical Statement: The authors are accountable for all aspects of the work in ensuring that questions related to the accuracy or integrity of any part of the work are appropriately investigated and resolved.

Open Access Statement: This is an Open Access article distributed in accordance with the Creative Commons Attribution-NonCommercial-NoDerivs 4.0 International License (CC BY-NC-ND 4.0), which permits the noncommercial replication and distribution of the article with the strict proviso that no changes or edits are made and the original work is properly cited (including links to both the formal publication through the relevant DOI and the license). See: https://creativecommons.org/licenses/by-nc-nd/4.0/.

\section{References}

1. International Agency for Research on Cancer, World Health Organization. Cancer today. Available online: https://gco.iarc.fr/today/home

2. Jemal A, Ward EM, Johnson CJ, et al. Annual Report to the Nation on the Status of Cancer, 1975-2014, Featuring Survival. J Natl Cancer Inst 2017;109:djx030.

3. European Association For The Study Of The Liver; European Organisation For Research And Treatment Of Cancer. EASL-EORTC clinical practice guidelines: management of hepatocellular carcinoma. J Hepatol 2012;56:908-43.

4. Llovet JM, Ricci S, Mazzaferro V, et al. Sorafenib in advanced hepatocellular carcinoma. N Engl J Med 2008;359:378-90.

5. Kudo M, Finn RS, Qin S, et al. Lenvatinib versus sorafenib in first-line treatment of patients with unresectable hepatocellular carcinoma: a randomised phase 3 noninferiority trial. Lancet 2018;391:1163-73.

6. Gordan JD, Kennedy EB, Abou-Alfa GK, et al. Systemic Therapy for Advanced Hepatocellular Carcinoma: ASCO Guideline. J Clin Oncol 2020;38:4317-45.

7. Finn RS, Qin S, Ikeda M, et al. Atezolizumab plus Bevacizumab in Unresectable Hepatocellular Carcinoma. N Engl J Med 2020;382:1894-905.

8. Zhuang BW, Li W, Xie XH, et al. Sorafenib versus hepatic arterial infusion chemotherapy for advanced hepatocellular carcinoma: a systematic review and meta-analysis. Jpn J Clin Oncol 2019;49:845-55.

9. Moher D, Liberati A, Tetzlaff J, et al. Preferred reporting items for systematic reviews and meta-analyses: the PRISMA statement. PLoS Med 2009;6:e1000097.

10. Lencioni R, Llovet JM. Modified RECIST (mRECIST) assessment for hepatocellular carcinoma. Semin Liver Dis 
2010;30:52-60.

11. Villaruz LC, Socinski MA. The clinical viewpoint: definitions, limitations of RECIST, practical considerations of measurement. Clin Cancer Res 2013;19:2629-36.

12. Jadad AR, Moore RA, Carroll D, et al. Assessing the quality of reports of randomized clinical trials: is blinding necessary? Control Clin Trials 1996;17:1-12.

13. Wells G, Shea B, O'Connell D, et al. The NewcastleOttawa Scale (NOS) for assessing the quality of nonrandomized studies in meta-analyses. Ottawa Hospital Research Institute, 2019. 2019. Available online: http:// ohri.ca/programs/clinical_epidemiology/oxford.asp

14. Tierney JF, Stewart LA, Ghersi D, et al. Practical methods for incorporating summary time-to-event data into metaanalysis. Trials 2007;8:16.

15. Higgins JP, Thompson SG, Deeks JJ, et al. Measuring inconsistency in meta-analyses. BMJ 2003;327:557-60.

16. Hiramine Y, Uto H, Imamura Y, et al. Sorafenib and hepatic arterial infusion chemotherapy for unresectable advanced hepatocellular carcinoma: A comparative study. Exp Ther Med 2011;2:433-41.

17. Jeong SW, Jang JY, Lee JE, et al. The efficacy of hepatic arterial infusion chemotherapy as an alternative to sorafenib in advanced hepatocellular carcinoma. Asia Pac J Clin Oncol 2012;8:164-71.

18. Nemoto T, Matsuda H, Nosaka T, et al. Comparison of hepatic arterial infusion chemotherapy and sorafenib in elderly patients with advanced hepatocellular carcinoma: A case series. Mol Clin Oncol 2014;2:1028-34.

19. Shiozawa K, Watanabe M, Ikehara T, et al. Comparison of Sorafenib and Hepatic Arterial Infusion Chemotherapy for Advanced Hepatocellular Carcinoma: A Propensity Score Matching Study. Hepatogastroenterology 2014;61:885-91.

20. Fukubayashi K, Tanaka M, Izumi K, et al. Evaluation of sorafenib treatment and hepatic arterial infusion chemotherapy for advanced hepatocellular carcinoma: a comparative study using the propensity score matching method. Cancer Med 2015;4:1214-23.

21. Kawaoka T, Aikata H, Hyogo H, et al. Comparison of hepatic arterial infusion chemotherapy versus sorafenib monotherapy in patients with advanced hepatocellular carcinoma. J Dig Dis 2015;16:505-12.

22. Kondo M, Morimoto M, Ishii T, et al. Hepatic arterial infusion chemotherapy with cisplatin and sorafenib in hepatocellular carcinoma patients unresponsive to transarterial chemoembolization: a propensity score-based weighting. J Dig Dis 2015;16:143-51.

23. Hatooka M, Kawaoka T, Aikata H, et al. Comparison of
Outcome of Hepatic Arterial Infusion Chemotherapy and Sorafenib in Patients with Hepatocellular Carcinoma Refractory to Transcatheter Arterial Chemoembolization. Anticancer Res 2016;36:3523-9.

24. Moriguchi M, Aramaki T, Nishiofuku H, et al. Sorafenib versus Hepatic Arterial Infusion Chemotherapy as Initial Treatment for Hepatocellular Carcinoma with Advanced Portal Vein Tumor Thrombosis. Liver Cancer 2017;6:275-86.

25. Terashima T, Yamashita T, Arai K, et al. Beneficial Effect of Maintaining Hepatic Reserve during Chemotherapy on the Outcomes of Patients with Hepatocellular Carcinoma. Liver Cancer 2017;6:236-49.

26. Yang H, Woo HY, Lee SK, et al. A comparative study of sorafenib and metronomic chemotherapy for Barcelona Clinic Liver Cancer-stage $C$ hepatocellular carcinoma with poor liver function. Clin Mol Hepatol 2017;23:128-37.

27. Kang MK, Park JG, Lee HJ. Comparison of clinical outcomes between sorafenib and hepatic artery infusion chemotherapy in advanced hepatocellular carcinoma: A STROBE-compliant article. Medicine (Baltimore) 2018;97:e0611.

28. Kodama K, Kawaoka T, Aikata H, et al. Comparison of clinical outcome of hepatic arterial infusion chemotherapy and sorafenib for advanced hepatocellular carcinoma according to macrovascular invasion and transcatheter arterial chemoembolization refractory status. J Gastroenterol Hepatol 2018;33:1780-6.

29. Lyu N, Kong Y, Mu L, et al. Hepatic arterial infusion of oxaliplatin plus fluorouracil/leucovorin vs. sorafenib for advanced hepatocellular carcinoma. J Hepatol 2018;69:60-9.

30. Moriya K, Namisaki T, Sato S, et al. Efficacy of bimonthly hepatic arterial infusion chemotherapy for advanced hepatocellular carcinoma. J Gastrointest Oncol 2018;9:741-9.

31. Ahn YE, Suh SJ, Yim HJ, et al. Comparison of Sorafenib versus Hepatic Arterial Infusion Chemotherapy-Based Treatment for Advanced Hepatocellular Carcinoma with Portal Vein Tumor Thrombosis. Gut Liver 2021;15:284-94.

32. Song DS, Song MJ, Bae SH, et al. A comparative study between sorafenib and hepatic arterial infusion chemotherapy for advanced hepatocellular carcinoma with portal vein tumor thrombosis. J Gastroenterol 2015;50:445-54.

33. Nakano M, Niizeki T, Nagamatsu H, et al. Clinical effects and safety of intra-arterial infusion therapy of cisplatin 
suspension in lipiodol combined with 5-fluorouracil versus sorafenib, for advanced hepatocellular carcinoma with macroscopic vascular invasion without extra-hepatic spread: A prospective cohort study. Mol Clin Oncol 2017;7:1013-20.

34. Choi JH, Chung WJ, Bae SH, et al. Randomized, prospective, comparative study on the effects and safety of sorafenib vs. hepatic arterial infusion chemotherapy in patients with advanced hepatocellular carcinoma with portal vein tumor thrombosis. Cancer Chemother

Cite this article as: Yan L, Lin J, Ke K, Wu Z, Huang J, Huang N, Yang W. A meta-analysis comparing hepatic arterial infusion chemotherapy and sorafenib for advanced hepatocellular carcinoma. Transl Cancer Res 2022;11(1):99-112. doi: $10.21037 /$ tcr-21-1839
Pharmacol 2018;82:469-78.

35. He M, Li Q, Zou R, et al. Sorafenib Plus Hepatic Arterial Infusion of Oxaliplatin, Fluorouracil, and Leucovorin vs Sorafenib Alone for Hepatocellular Carcinoma With Portal Vein Invasion: A Randomized Clinical Trial. JAMA Oncol 2019;5:953-60.

36. Liu M, Shi J, Mou T, et al. Systematic review of hepatic arterial infusion chemotherapy versus sorafenib in patients with hepatocellular carcinoma with portal vein tumor thrombosis. J Gastroenterol Hepatol 2020;35:1277-87. 


\section{PubMed}

((((((Carcinoma, Hepatocellular[MeSH Terms]) OR (hepatocellular carcinoma)) OR (HCC)) OR (hepatocarcinoma)) OR (liver cancer)) OR (liver cell carcinoma)) AND (((((hepatic arterial infusion chemotherapy) OR (hepatic artery infusion chemotherapy)) OR (hepatic arterial infusion)) OR (hepatic artery infusion)) OR (HAIC)) OR (HAI))) AND (((sorafenib[MeSH Terms]) OR (sorafenib)) OR (nexavar)) Filters: English

\section{Web of Science}

(TOPIC: (hepatocellular carcinoma) OR TOPIC: (HCC) OR TOPIC: (hepatocarcinoma) OR TOPIC: (liver cancer) OR TOPIC: (liver cell carcinoma)) AND (TOPIC: (hepatic arterial infusion chemotherapy) OR TOPIC: (hepatic artery infusion chemotherapy) OR TOPIC: (hepatic arterial infusion) OR TOPIC: (hepatic artery infusion) OR TOPIC: (HAIC) OR TOPIC: (HAI) ) AND (TOPIC: (sorafenib) OR TOPIC: (nexavar) ) Refined by: LANGUAGES: ( ENGLISH)

\section{Embase}

((hepatocellular AND carcinoma OR hcc OR hepatocarcinoma OR (liver AND cancer) OR (liver AND cell AND carcinoma))) AND (hepatic AND arterial AND infusion AND chemotherapy OR (hepatic AND artery AND infusion AND chemotherapy) OR (hepatic AND arterial AND infusion) OR (hepatic AND artery AND infusion) OR haic OR hai) AND (sorafenib OR nexavar) AND [english]/lim

\section{COCHRANE}

((hepatocellular carcinoma):ti,ab,kw OR (HCC):ti,ab,kw OR (hepatocarcinoma):ti,ab,kw OR (liver cancer):ti,ab,kw OR (liver cell carcinoma):ti,ab,kw ) AND ((hepatic arterial infusion chemotherapy):ti,ab,kw OR (hepatic arterial infusion):ti,ab,kw OR (HAIC):ti,ab,kw OR (HAI):ti,ab,kw) AND ((sorafenib):ti,ab,kw OR (nexavar):ti,ab,kw) 